\title{
Early diagnosis and treatment of invasive aspergillosis as a main determinant of outcome - review of literature according to the presented case report
}

\author{
Michał Borys ${ }^{1}$, Paweł Piwowarczyk¹, Justyna Sysiak¹, Mirosław Czuczwar ${ }^{1}$, Andrzej Prystupa² \\ 1 II Department of Anaesthesiology and Intensive Care, Medical University, Lublin, Poland \\ 2 Department of Internal Medicine, Medical University of Lublin, Poland
}

Borys M, Piwowarczyk P, Sysiak J, Czuczwar M, Prystupa A. Early diagnosis and treatment of invasive aspergillosis as a main determinant of outcome - review of literature according to the presented case report. Ann Agric environ Med. 2017 ; 24(1): $100-103$. doi: $10.5604 / 12321966.1233966$

\begin{abstract}
Although. Aspergillus spp infection is not the major cause of morbidity in Intensive Care Units (ICUs), mortality among patients treated for it is tremendous. Moreover, invasive aspergillosis (IA) is an independent risk factor of hospital costs and length of stay. The prevalence of this disease is inversely correlated with the immunocompetence of individuals; for instance, the incidence of IA among patients with leukemia is estimated as high as $12.7 \%$. Although there is a significant improvement in the antifungal armamentarium, the appropriate treatment is still being given too late, mostly because of late diagnosis. As well as the diagnosis, the criteria for recognition of IA constitute a challenge.

Objective. The aim of this review, based on a case report, is to introduce the problem of poor diagnosis and treatment of $I A$, especially in the critical care settings. The presented scenario is an example which assists in showing the evidence-based medicine (EBM) approach to the treatment of fungal infections. Furthermore, to demonstrate the appropriate approach to diagnosis and treatment of invasive aspergillosis, the guidelines of The European Organization for Research and Treatment of Cancer/Mycoses Study Group (EORTC/MSG) are presented.

Conclusions. According to presented literature, Galactomannan assay enables early diagnosis and remains a specific and sensitive tool to diagnose Asppergillosis, both in serum and BAL fluid. The guidelines recommend voriconazole as a first line treatment in IA. Failure to detect and implement proper antifungal treatment may lead to fatal consequences, as in the presented case.
\end{abstract}

\section{Key words}

immunodeficiency, invasive aspergillois, ICU, galactomannan, voriconazole

\section{INTRODUCTION}

Invasive aspergillosis (IA) is becoming an increasing challenge in Intensive Care Units (ICUs) in both Europe and the United States. ICU physicians should be aware of IA-attributable mortality, which varies from $35 \%-46 \%$ in haematooncological malignancies and haematopoietic stem cell transplantation (HSCT) recipients [1, 2, 3, 4]. Moreover, IA is an independent risk factor for increased hospital costs and increased length of stay (LOS) $[4,5]$.

Constantly increasing costs in many healthcare systems worldwide are associated with the implementation of the latest drugs, diagnostic techniques and invasive procedures. The progress in medicine is undoubtedly beneficial, yet one of the recently most discussed issues of the potential risks of increased invasiveness in all fields of medical specialties is the increasing rate of fungal infections [6]. For instance, allergic bronchopulmonary Aspergillosis is the most common form of disease because of ubiquitous presence of this fungus [7]. On the other hand, the prevalence of IA is inversely correlated with the immunocompetence of individuals. Patients suffering from haemopoetic malignancies and solid

Address for correspondence: Andrzej Prystupa, Department of Internal Medicine Medical University of Lublin.

E-mail:aprystup@mp.pl

Received: 2 December 2014; accepted: 29 July 2015; first published on February 2017 organ transplantation receivers are at the highest risk of Aspergillosis $[8,9]$. The incidence of IA among patients with leukemia is estimated to be as high as $12.7 \%$ [9]. Another group of patients highly susceptible to IA are those treated in ICUs, where the prevalence of this invasive fungal disease in non-malignant patients is estimated to be as high as 7\% [10].

Despite a significant improvement in the antifungal armamentarium, the usual hospital practice shows that an appropriate treatment is given too late, mostly because of the delayed diagnosis [11]. Traditional diagnostic procedures, such as cultures or x-rays, show both the lack of sensitivity and specificity [12]. However, modern diagnostic methods, e.g. computed tomography (CT) imaging, are not always feasible for ICU patients or are not well validated $(1,3-\beta$-Dglucan assay) in IA $[12,13]$. Another serious problem is the criteria for detecting an IA. Since the prevalence of Candida spp infections is much higher than those associated with Aspergillus spp, the majority of diagnostic tools used to aid the clinicians in diagnosing fungal infections are tailored to detect yeast incidences. The aim of this review is to identify the most common problems associated with an IA in the ICU setting. The presented case report highlights shortcomings in clinical practice. 


\section{CASE REPORT}

On 12 March 2013, a 61-year-old female was admitted to the ICU in a tertiary academic hospital. She initially presented with a bilateral pneumonia complicated by an acute respiratory failure and cardiovascular failure [Fig. 1]. The patient died the day after admission to the ICU, and the autopsy revealed IA.

Three years before admission to the ICU the patient was diagnosed with Chronic Leukemic Leukemia (CLL) in the second stage according to RAI. Despite sequential treatment, full recovery was not achieved, and progress of CLL resulted in a subsequent admission to the haematooncology ward. During the last four months, the patient was hospitalized three times due to fever of unknown origin. Each time, broad-spectrum antibiotics were implemented (i.e. amikacin, ciprofloxacine, ceftezidime, amoxicillin/clavulanic acid, imipenem with cilastatin), and increased doses of steroids were administered (i.e. prednisone, methyloprednisone and dexamethasone). The antifungal agents included in empiric treatment were fluconazole, ketoconazole and a short-course treatment with itraconazole. All collected blood cultures were negative. The only CT scan performed during the deterioration of the state of the patient revealed nodular thickening of the peribronchovascular interstitium and interlobular septa, thus lymphangitis carcinomatosa was diagnosed. After admission to the ICU, antifungal and broadspectrum antibiotic therapy was initiated (caspofungin and imipemem with cilastatin). BAL and blood samples were collected towards ELISA galactomannan and mannan tests, as well as for the growth of cultures. Despite the treatment, the patient died the next day. The direct microscopy samples obtained from BAL revealed Aspergillosis.

\section{DISCUSSION}

This case report is a description of therapeutic and diagnostic process in the course of an IA which took place in a tertiary hospital. Recent clinical and scientific achievements in the therapy and diagnosis of Aspergillosis are presented. During the review, problems and doubts in the patient's therapeutic process are emphasized. Furthermore, to demonstrate the appropriate approach to diagnosis and treatment of IFD, the Guidelines of The European Organization for Research and Treatment of Cancer/Mycoses Study Group (EORTC/MSG) are presented. In 2008, EORTC/MSG published the revised definitions of invasive fungal disease (IFD) [11]. According to this definition, three categories of IFD are presented: 'proven', 'probable' and 'possible' IFD.

Diagnostic methods to prove fungal infection. The definition for proven IFD requires an identification of the fungal elements, and IA needs the conformation with cultures or a histopathological examination. Detecting fungal elements from a normally sterile environment reaffirms IFD [11]. Obtaining specimens for cultures is the most traditional approach to detection of infectious diseases [14], and creates the opportunity not only for the recognition of specific genus, but also enables testing the susceptibility prior to an initiation of antifungal treatment [15]. Before the era of galactomannan evaluation, cultures were the main test for a conformation of fungal infection [15]. However, there are several problems applicable to culture results and collection. A positive culture from a normally sterile site, e.g. pleural fluid, confirms a fungal infection if linked with clinical features [11]. In contrast, the same result obtained from bronchoalveolar lavage (BAL) fluid or urine, without any signs of infection, reveals a fungal colonization. Blood culture growth is interpreted as a fungal contamination. Moreover, the sensitivity of cultures obtained from the respiratory tract does not exceed $50 \%[16,17,18]$. Torelli et al. compared the real-time PCR (polymerase chain reaction) assay with the GM test and cultures for the detection of Aspergillus species [19]. All specimens were obtained from BAL. Both the PCR and GM tests presented the same, $94.1 \%$ accuracy in patients with proven/probable IA. In contrast, only $41 \%$ cultures were positive among this group of patients.

The question is, whether there is any necessity to wait for the result of traditional culture if it shows the lack of sensitivity in detecting IA? The answer is not completely obvious. The induction of antifungal treatment should not be based only on diagnostic tests, especially cultures, because of possible colonization [20]. However, this traditional approach enables the testing of susceptibility, and expands the number of species possible to reveal, as far as possible, a basis for others tests [21]. Furthermore, the results of positive cultures may distinguish the difference between an active disease, while viable fungi can be found, and the period after the initiation of treatment, when the GM assay may still be positive [22].

The other approach to confirm proven IA is a biopsy of tissue for histopathological examination [11]. The problem is that a biopsy is an invasive procedure, and the risk between an injury and potential benefits should be considered. For that reasons, such an intervention is limited in questionable cases [23]. The standard staining with haematoxylin and eosin is mostly sufficient for the detection of Aspergillus hyphae [24]. However, the material obtained with a biopsy requires cultures or the PCR assay to recognize the exact fungal genus [25].

Probable invasive fungal disease. The category of probable IFD (EORTC/MSG) is composed of three elements: host factors, clinical and mycological criteria. All of these elements must be included to recognize probable IFD [11]. The host factors concern severely immunocompromised patients. All host-dependent components are shown in Table 1. Some inherited, but rather rare conditions, such as chronic granulomatous disease (prevalence 1/300 000 births), are well known risk factors of IA [26]. In contrast, many patients are at risk of fungal infections due to a therapeutic process,

Table 1. Host factors, according to the EORTC/MSG definitions [De Pauw 2008]

i. Recent history of neutropenia $(<0.5 \times 109$ neutrophils/L $[<500$ neutrophils/ $\mathrm{mm}^{3}$ ] for $>10$ days) temporally related to the onset of fungal disease.

ii. Receipt of an allogeneic stem cell transplant.

iii. Prolonged use of corticosteroids (excluding among patients with allergic bronchopulmonary aspergillosis) at a mean minimum dose of $0.3 \mathrm{mg} / \mathrm{kg} /$ day of prednisone equivalent for $>3$ weeks.

iv. Treatment with other recognized T cell immunosuppressants, such as cyclosporine, TNF-a blockers, specific monoclonal antibodies (such as alemtuzumab), or nucleoside analogues during the previous 90 days.

v. Inherited severe immunodeficiency (such as chronic granulomatous disease or severe combined immunodeficiency). 
for example: immunotherapy or prolonged corticosteroids administration. These patients need to be carefully evaluated, and healthcare providers retain vigilant. The appropriate diagnostic tools should be employed and the treatment established whenever there is a threat of fungal infection.

Furthermore, EORTC/MSG reports that a mean minimum dose of $0.3 \mathrm{mg} / \mathrm{kg} /$ day of prednisone, or its equivalent, given for a period of more than three weeks, is related to increased risk of IFD [11]. Many studies present positive correlation between the dose and duration of steroid administration and the risk of IA $[27,28,29,30]$. A prednisone dose higher than $1 \mathrm{mg} / \mathrm{kg} /$ day increases the chance of morbidity due to Aspergillosis [30]. O'Donnell et al. demonstrated that an increased dose of prednisone $(0.25$ compared to $0.5-$ $1 \mathrm{mg} / \mathrm{kg} /$ day) was correlated with six-fold higher risk of IA [28]. Consequently, corticosteroids diminish clinical signs of infection, e.g. fever, and cough, as well as attenuate some laboratory parameters: $\mathrm{C}$ - reactive protein, white blood count. [27]; therefore, the recognition of IA may be delayed. Many other patterns of fungal infection can be damped in steroid-treated patients compared to neutropenic individuals [31]. These include: TNF- $\alpha$, IL-10, galactomannan, a number of conidia and hyphae. The histological features of IA are also changed, which influences chest X-ray and CT imaging [32]. The typical radiographic picture of pulmonary Aspergillosis in neutropenic patients includes the 'halo sign', a nodular lesion surrounded by ground-glass opacity. Corticosteroids alter this image. There is no standard radiological description of IA in steroid-treated patients, thus the chest imaging is not such a helpful tool as it might seem to distinguish pulmonary Aspergillosis from other conditions [32].

The galactomannan (GM) and 1,3- $\beta$-D-glucan assays are well-known for detecting IFD $[13,18,33,34,35]$. The former is both more specific for IA and strongly correlates with the outcome of patients $[18,33,35]$, whereas the latter is not a specific instrument for detecting Aspergillus genus, but can be useful for monitoring the severity of infection if IA is recognized [13]. The GM assay is a specific and sensitive tool for diagnosing Asppergillosis, both in serum and BAL fluid $[18,35,36]$. This test is not well validated for detecting Asppergillosis in other fluids, e.g. urine and sputum [37]. There are also consecutive limitations: the GM is not completely specific for Aspergillus genus, and other fungi like Penicillium, Fusarium, Alternaria, and Histoplasma can be revealed by this method [38]. Furthermore, one of the most popular antibiotics used in the ICU (piperacillintazobactam), and electrolyte solutions (e.g. Plasmalyte), may interfere with the test results (false positive) $[39,40]$.

Fever and possible invasive fungal disease. According to the EORTC/MSG definitions of IFD, fever is not a host factor, and due to the lack of specificity is only considered a clinical sign [41]. However, persistent fever, in particular when antibiotics are used ineffectively, can often suggest a fungal infection [41, 42]. In such case, if clinical signs and host factors are present, without the conformation in mycological tests, the criteria of possible IFD are fulfilled. With the lack of mycological test, especially indirect ones, criteria for detecting possible IFD could have been taken into consideration. Thus, in this situation, an insightful investigation of the patient should have been carried out instead of subsequent administration of antibiotics.
Antifungal treatment. In the critical care setting, voriconazole is the drug of choice when invasive pulmonary Aspergillosis is presumed [43]. The alternative agents comprise: itraconazole, posaconazole, amphotericin B and echinocandins (i.e. micafungin, anidulafungin and caspofungin). Voriconazole shows a superior effectiveness over amphotericin B deoxycholate (AmB-D) for the treatment of IA [44]. The initial dose of voriconazole is $6 \mathrm{mg} / \mathrm{kg}$ (repeated after $12 \mathrm{~h}$ ), then $4 \mathrm{mg} / \mathrm{kg}$ given every 12 hours. Both mould-active azoles (i.e. itraconazole, posaconazole) have the same limitation; the only available route of administration is oral one. While voriconazole shows lack of efficacy, caspofungin is regarded as a salvage therapy for IA [45].

Shortcomings in the clinical approach. In the presented case, some problems should be highlighted. Firstly, no fungal specific tests were performed before admission to the ICU. These tests were available in the hospital (i.e. GM and 1,3- $\beta$-Dglucan assays). Specimens for cultures were only taken from the patient's blood. Even if the blood culture growth result had revealed Aspergillus spp, that would only have been interpreted as a fungal contamination [11]. No biopsy or samples from the respiratory tract were taken untill the ICU admission. Secondly, three different steroids were given at the same time (methylprednisolone, dexamethasone and prednisone). During the final two weeks, a total of $1,000 \mathrm{mg}$ of methylprednisolone was administered daily - the equivalent to $1,250 \mathrm{mg}$ of prednisone. An increased dose of prednisone is an independent risk factor of IA $[11,28]$. The reason of noncharacteristic CT scans were probably the steroid-treatment (lack of halo sign typical for neutropenic patients). This was probably the reason why lymphangitis carcinomatosa was recognized.

The patient received myriad antibacterial agents from different groups, but antifungal prophylaxis covered only Candidia spp. (itraconazole was given for only a few days). Moreover, until admission to the ICU, fluconazole was the only antifungal prophylaxis applied. In the ICU, multiple organ failure was diagnosed, and because of suspected IFD, treatment with intravenous caspofungin was introduced.

\section{CONCLUSIONS}

The presented case demonstrates the lack of compliance with the EORTC/MSG definitions of IFD among healthcare providers. The available diagnostic tools and therapeutic options create opportunities to manage IA effectively. Further-going scientific development is not a substitute for knowledge and clinical vigilance. Failure to detect and implement proper antifungal treatment may lead to fatal consequences, as in the presented case.

\section{REFERENCES}

1. Pfaller MA, Diekema DJ. Epidemiology of invasive Candidiasis: a persistent public health problem. Clin Microbiol Rev. 2007; 20:133-63.

2. Neofytos D, Horn D, Anaissie E, et al. Epidemiology andoutcome of invasive fungal infection in adult hematopoietic stem cell transplant recipients: analysis of multicenter Prospective Antifungal Therapy (PATH) alliance registry, Clin Infect Dis. 2009; 48: 265-73.

3. Nicolle MC, Benet T, Thiebaut A et al. Invasive aspergillosis in patients with hematologic malignancies: incidence and description of 127 cases 
enrolled in a single institution prospective survey from 2004 to 2009 , Haematologica. 2011; 96: 1685-91.

4. Baddley JW, Stephens JM, Ji X, Gao X, Schlamm HT, Tarallo M. Aspergillosis in Intensive Care Unit (ICU) patients: epidemiology and economic outcomes BMC Infect Dis. 2013; 13: 29.

5. Kim A, Nicolau DP, Kuti JL. Hospital costs and outcomes among intravenous antifungal therapies for patients with invasive aspergillosis in the United States, Mycoses. 2011; 54: 301-12.

6. Wisplinghoff $\mathrm{H}$, Bischoff T, Tallent SM, et al. Nosocomial bloodstream infections in US hospitals: analysis of 24,179 cases from a prospective nationwide surveillance study. Clin Infect Dis. 2004; 39: 309-17.

7. De Lucca AJ, Boué SM, Carter-Wientjes CH, Bland JM, Bhatnagar $\mathrm{D}$, Cleveland TE. Volatile profiles of toxigenic and non-toxigenic Aspergillus flavus using SPME for solid phase extraction. Ann Agric Environ Med. 2010; 17: 301-8.

8. Hidron AI, Edwards JR, Patel J, et al. Antimicrobial-resistant pathogens associated with healthcare-associated infections: annual summary of data reported to the national healthcare safety network at the Centers for Disease Control and Prevention, 2006-2007. Infect Control Hosp Epidemiol. 2008; 29: 996-1011.

9. Chamilos G, Luna M, Lewis RE, et al. Invasive fungal infections in patients with hematologic malignancies in a tertiary care cancer center: an autopsy study over a 15-year period (1989-2003). Haematologica. 2006; 91: 986-9.

10. Meersseman W, Lagrou K, Maertens J, et al. Galactomannan in bronchoalveolar lavage fluid: a tool for diagnosing aspergillosis in intensive care unit patients, Am J Resp Crit Care Med. 2008; 177: 27-34.

11. De Pauw B, Walsh TJ, Donnelly JP, Stevens DA, Edwards JE, et al. Revised Definitions of Invasive Fungal Disease from the European Organization for Research and Treatment of Cancer/Invasive Fungal Infections Cooperative Group and the National Institute of Allergy and Infectious Diseases Mycoses Study Group (EORTC/MSG) Consensus Group. Clin Infect Dis. 2008; 46: 1813-21.

12. Tsitsikas DA, Morin A, Araf S, Murtagh B, Johnson G, Vinnicombe S, et al. Impact of the revised (2008) EORTC/MSG definitions for invasive fungal disease on the rates of diagnosis of invasive aspergillosis. Med Mycol. 2012; 50: 538-42.

13. Ostrosky-Zeichner L, Alexander BD, Kett DH, et al. Multicenter clinical evaluation of the $(1 \rightarrow 3) \beta$-D-glucan assay as an aid to diagnosis of fungal infections in humans. Clin Infect Dis. 2005; 41: 654-9.

14. Leslie CE, Flannigan B, Milne LJR. Morphological studies on clinical isolates of Aspergillus fumigatus. J Med Vet Mycol. 1988; 26: 335-41.

15. Horvath JA, Dummer S. The use of respiratory-tract cultures in the diagnosis of invasive pulmonary aspergillosis. Am J Med. 1996; 100: 171-8.

16. Levine SJ. An approach to the diagnosis of pulmonary infections in immunosuppressed patients. Semin Respir Infect. 1992; 7: 81-95.

17. Bergeron A, Belle A, Sulahian A, et al. Contribution of galactomannan antigen detection in BAL to the diagnosis of invasive pulmonary aspergillosis in patients with hematologic malignancies. Chest. 2010; 137: 410-15.

18. Maertens J, Maertens V, Theunissen K, et al. Bronchoalveolar lavage fluid galactomannan for the diagnosis of invasive pulmonary aspergillosis in patients with hematologic diseases. Clin Infect Dis. 2009, 49: 1688-93.

19. Torelli R, et al. 2011. Diagnosis of invasive aspergillosis by a commercial real-time PCR assay for Aspergillus DNA in bronchoalveolar lavage fluid samples from high-risk patients compared to a galactomannan enzyme immunoassay. J Clin Microbiol. 2011; 49: 4273-8.

20. Luong ML, Clancy CJ, Vadnerkar A, et al. Comparison of an aspergillus real-time polymerase chain reaction assay with galactomannan testing of bronchoalvelolar lavage fluidfor the diagnosis of invasive pulmonary aspergillosis in lung transplant recipients. Clin Infect Dis. 2011; 52: 1218-26.

21. Balajee SA, Gribskov JL, Hanley E, Nickle D, Marr A. Aspergillus lentulus sp. nov., a new sibling species of A. fumigatus. Eukaryotic Cell. 2005; 4:625-32.

22. Walsh TJ, Wissel MC, Grantham KJ, et al. Molecular detectionand species-specific identification of medically important Aspergillus species by real-time PCR in experimental invasive pulmonary aspergillosis. J Clin Microbiol. 2011; 49: 4150-7.

23. Riwes MM, Wingard JR. Diagnostic methods for invasive fungal diseases in patients with hematologic malignancies. Expert Rev Hematol. 2012; 5: 661-9.
24. Denning DW, Kibbler CC, Barnes RA. British Society for Medical Mycology proposed standards of care for patients with invasive fungal infections. Lancet Infect Dis. 2003; 3: 230-40.

25. Paterson PJ, Seaton S, McHugh TD, et al. Validation and clinical application of molecular methods for the identification of molds in tissue. Clin Infect Dis. 2006; 42: 51-56.

26. Kang EM, Marciano BE, DeRavin SS, Zarember K, Holland SM, Malech HL. Chronic Granulomatous Disease: Overview and Hematopoietic Stem Cell Transplant. J Allergy Clin Immunol. 2011; 127: 1319-26.

27. Lionakis MS, Kontoyiannis DP. Glucocorticoids and invasive fungal infections. Lancet. 2003; 362: 1828-38.

28. O'Donnell MR, Schmidt GM, Tegtmeier BR, et al. Prediction of systemic fungal infection in allogeneic marrow recipients: impact of amphotericin prophylaxis in high-risk patients. J Clin Oncol. 1994; 12: 827-34.

29. Grow WB, Moreb JS, Roque D, et al. Late onset of invasive Aspergillus infection in bone marrow transplant patients at a university hospital. Bone Marrow Transplan. 2002; 29: 15-19.

30. Gustafson TL, Schaffner W, Lavely GB, et al. Invasive aspergillosis in renal transplant recipients: correlation with corticosteroid therapy. J Infect Dis. 1983; 148: 230-8.

31. Balloy V, Huerre M, Latge JP, Chignard M. Differences in patterns of infection and inflammation for corticosteroid treatment and chemotherapy in experimental invasive pulmonary aspergillosis. Infect Immun. 2005; 73: 494-503.

32. Greene RE, Schlamm HT, Oestmann JW, et al. Imaging findings in acute invasive pulmonary aspergillosis: clinical significance of the halo sign. Clin Infect Dis. 2007; 44: 373-9.

33. Salonen J, Lehtonen OP, Teräsjärvi MR, Nikoskelainen J. Aspergillus antigen in serum, urine and bronchoalveolar lavage specimens of neutropenic patients in relation to clinical outcome, Scand J Infect Dis. 2000 ; 32: 485-90.

34. Obayashi T, Yoshida M, Mori T, et al. Plasma $(1 \rightarrow 3)$ - $\beta$-D-glucan measurement in diagnosis of invasive deep mycosis and fungal febrile episodes. The Lancet. 1995; 345: 17-20.

35. Miceli MH, Grazziutti ML, Woods G, et al. Strong correlation between serum Aspergillus galactomannan index and outcome of aspergillosis in patients with hematological cancer: clinical and research implications. Clin Infect Dis. 2008; 46: 1412-22.

36. Maertens J, Maertens V, Theunissen K, et al. Bronchoalveolar lavage fluid galactomannan for the diagnosis of invasive pulmonary aspergillosis in patients with hematologic diseases. Clin Infect Dis. 2009; 49: 1688-93.

37. Klont RR, Mennink-Kersten MASH, Verweij PE. Utility of Aspergillus antigen detection in specimens other than serum specimens. Clin Infect Dis. 2004; 39: 1467-74.

38. Swanink CMA, Meis JFGM, Rijs AJMM, Donnelly JP, Verweij JP. Specificity of a sandwich enzyme-linked immunosorbent assay for detecting Aspergillus galactomannan. J Clin Microbiol. 1997. 35: 257-60.

39. Sulahian A, Touratier S, Ribaud P. False positive test for aspergillus antigenemia related to concomitant administration of piperacillin and tazobactam. N Engl J Med. 2003; 349: 2366-7.

40. Racil Z, Kocmanova I, Lengerova M, Winterova J, Mayer J. Intravenous PLASMA-LYTE as a major cause of false positive results of platelia Aspergillus test for galactomannan detection in serum. J Clin Microbiol. 2007; 45: 3141-2.

41. Sherif R, Segal BH. Pulmonary Aspergillosis: clinical presentation, diagnostic tests, management and complications. Curr Opin Pulm Med. 2010; 16: 242-50.

42. Delisle MS, Williamson DR, Albert M, Perreault MM, Jiang X, Day AG et al. Impact of Candida species on clinical outcomes in patients with suspected ventilator-associated pneumonia Can Respir J. 2011; 18: $131-6$.

43. Walsh TJ, Anaissie EJ, Denning DW, et al. Treatment of aspergillosis: clinical practice guidelines of the Infectious Diseases Society of America. Clin Infect Dis. 2008; 46: 327-60.

44. Herbrecht R, Denning DW, Patterson TF, Bennett JE, Greene RE, Oestmann JW, Kern WV, Marr KA, Ribaud P, Lortholary O, et al. Voriconazole versus amphotericin B for primary therapy of invasive aspergillosis. N Engl J Med. 2002; 347: 408-15.

45. Maertens J, Raad I, Petrikkos G, Boogaerts M, Selleslag D, Petersen FB et al. Efficacy and safety of caspofungin for treatment of invasive aspergillosis in patients refractory to or intolerant of conventional antifungal therapy. Clin Infect Dis. 2004; 39: 1563-71. 